\title{
The data and read/write controller for March-based SRAM diagnostic algorithm MBIST
}

\begin{abstract}
This paper presents the implementation of March-based algorithm as proposed in into an Memory Built-in Self-Test (MBIST) data and read/write controller. The design uses the approach of Finite State Machine (FSM)-based architecture which is more plausible since the design was part of the engine that will be exclusively developed for the testing of this algorithm alone. This controller will represent a portion of MBIST engine that can be incorporated together with other portions to build a complete MBIST engine.
\end{abstract}

Keyword: Finite state machine (FSM); Functional fault models (FFMs); March algorithm; Memory built in self test (MBIST); Stuck-at faults (SAF) 